\title{
Estresse pós-traumático da criança sobrevivente de câncer e sua percepção acerca da experiência parental
}

\author{
Camila Tokarski Boaventura \\ Instituto Nacional de Câncer \\ Tereza Cristina Cavalcanti Ferreira de Araujo \\ Universidade de Brasilia
}

\begin{abstract}
Resumo
Nas últimas décadas, paralelamente ao aumento progressivo das taxas de cura em Oncologia Pediátrica, evidencia-se um interesse crescente pela sobrevivência ao câncer na infância. Esta investigação teve como objetivo analisar essa experiência de sobrevivência ao câncer na infância, enfatizando a percepção da criança acerca da experiência parental e avaliando o Transtorno de Estresse Pós-traumático infantil nesse contexto. Realizaram-se entrevistas semiestruturadas com a criança, complementadas pela aplicação do UCLA Posttraumatic Stress Disorder Reaction Index for DSM-IV - Child Version. Evidenciaram-se taxas moderadas de diagnóstico total e parcial de Transtorno de Estresse Pós-Traumático. Especificamente no que se refere aos sintomas de estresse pós-traumático, os escores encontrados foram baixos. Os resultados também sugeriram uma relação entre o modo como a experiência parental é percebida pela criança e o escore total de gravidade de Transtorno de Estresse Pós-traumático Infantil. Recomendam-se estudos com amostras mais extensas, visando disponibilizar instrumentos de avaliação para a área da saúde.
\end{abstract}

Palavras-chave: sobrevivência; câncer; criança; estresse pós-traumático infantil; psico-oncologia pediátrica.

\begin{abstract}
Post-Traumatic stress disorder of the child survivor of cancer and their perception about parental experience. This investigation aimed to describe, analyze and comprehend the experience of survival after childhood cancer, emphasizing the child's perception of the parent's experience and the evaluation of the child PostTraumatic Stress Disorder. So, semi-structured interviews were conducted with the participants, who also answered the UCLA Posttraumatic Stress Disorder Reaction Index for DSM-IV-Child Version. The child's perception of the parental experience confirmed evidences pointed out by specialized literature. The total and partial diagnosis rates of Post-Traumatic Stress Disorder were moderate. Finally, it was possible to suggest a likely connection between the way the parental experience is perceived and the total severity score of the Post-Traumatic Stress Disorder in children. Further investigation, with more significant samples, is recommended in order to develop evaluation tools to be applied in other health situations.
\end{abstract}

Keywords: survival; cancer; child; child post-traumatic stress; pediatric psycho-oncology.

A partir da década de 1960, constata-se um crescente aumento no número de sobreviventes ao câncer na infância. Nos países desenvolvidos, as taxas de incidência são crescentes ou estáveis, mas a mortalidade é decrescente. De fato, sabe-se que as taxas de sobrevida atingiram $75 \%$ na última década (Andrea, 2008; Braga, Latorre, \& Curado, 2002; Perina, Mastellaro, \& Nucci, 2008; Roth, Holland, \& Murillo, 2006). Já nos países em desenvolvimento, tais tendências ainda são em grande parte desconhecidas. Mas, cabe destacar que os indicadores podem variar significativamente em um mesmo país, o que exige esforços contínuos das redes de saúde para diminuir diferenças regionais ocasionadas pela dificuldade de acesso a serviços de qualidade (Coleman et al., 2008).

Neste contexto, diversos trabalhos acerca da sobrevivência ao câncer na infância foram publicados desde a década de
1980, impulsionados, sobretudo, pelos desafios assistenciais enfrentados pelas equipes de saúde, e particularmente pelos psicólogos envolvidos com o acompanhamento de crianças e adolescentes após a alta do tratamento. Salientavam-se, notadamente, as dificuldades de integração da noção de cura pelo próprio paciente e por seus familiares (Alapetite et al., 1988; Alby, 1988; Lozowski, 1993; Pucheu, 1988).

Diante destas constatações clínicas, muitas pesquisas foram feitas visando avaliar a adaptação psicológica destes sobreviventes e investigar possíveis sequelas psicológicas e sociais. Dentre as variáveis privilegiadas nestes trabalhos, destacaram-se: depressão, ansiedade, desempenho acadêmico, autoestima, distúrbios comportamentais, inserção profissional, relações conjugais e familiares, cuidados com a saúde e projeto de vida (Dahlquist, Czyzewski, \& Jones, 1996; Eiser \& Havermans, 
1994; Frisch \& Desmarez, 1988; Mulhern, Fairclough, Smith, \& Douglas, 1992; Nicholson, Fears, \& Byrne, 1994).

Araujo e Arrais (1998) compararam as conclusões de estudos até então realizados e notaram disparidade nos resultados obtidos para as principais variáveis pesquisadas. Segundo as autoras, tal limitação explicava-se, em parte, pelas dificuldades inerentes à noção de cura e sobrevivência. Desta maneira, não havia homogeneidade de critérios adotados nos estudos para constituição das amostras, delineamento metodológico e análise dos dados. Neste mesmo sentido, o Comitê Pediátrico da Sociedade Brasileira de Psico-Oncologia (2000), em consonância com a Sociedade Internacional de Oncologia Pediátrica, traçou diretrizes para a assistência aos pacientes curados, defendendo a promoção da saúde física, psicossocial e socioeconômica.

\section{Estresse pós-traumático}

Segundo Janoff-Bulman (1999), logo após uma experiência aversiva, o indivíduo entra em um estado de desintegração psicológica, no qual pouco sente ou reconhece da ameaça representada pelo trauma. Ou seja, negação e entorpecimento caracterizam sua condição. Em caso de traumas graves, as estratégias usuais de recuperação psicológica podem não mais ser eficazes, sendo necessário um período de descanso em que a pessoa viva de forma a exigir pouco de seu self (Baumeister, Faber, \& Wallace, 1999). Ora, a experiência oncológica é permeada por múltiplas situações aversivas, sendo que para Alderfer, Labay e Kazak (2003), o modelo de estresse póstraumático captura a natureza traumática do câncer infantil.

Apesar dos estudos anteriores terem focalizado a adaptação psicológica e a identificação de sequelas físicas e psicológicas, as pesquisas passaram, efetivamente, a avaliar riscos de estresse pós-traumático entre sobreviventes ao câncer e seus familiares após a inclusão dessa experiência como evento traumático pela American Psychiatric Association (2000) no Manual Diagnóstico e Estatístico de Desordens Mentais, DSM-IV, em 1994. Assim, a partir de 1996, percebe-se uma forte tendência para a condução de investigações sobre Transtorno de Estresse Pós-Traumático - TEPT (Resende \& Araujo, 1999).

Vale lembrar que o TEPT apresenta duas características principais, que são o evento traumático, definido como "exposição a um evento que envolva a ocorrência ou a ameaça consistente de morte ou ferimentos graves para si ou para outros, associada a uma resposta intensa de medo, desamparo, ou horror" (Figueira \& Mendlowicz, 2003, p. 12); e as três dimensões de sintomas que se desenvolvem a partir desse evento, ou seja: a) o evento traumático é persistentemente revivido (ex.: pesadelos, recordações aflitivas, recorrentes e intrusivas); b) esquiva persistente de estímulos associados ao trauma e entorpecimento da responsividade geral; e c) sintomas persistentes de excitabilidade aumentada, incluindo hipervigilância, dificuldades de concentração e irritabilidade.

Mais recentemente, outros estudos internacionais averiguaram a incidência de altas taxas de sintomas de estresse pós-traumático entre sobreviventes ao câncer na infância, seus pais e irmãos; apesar das taxas de ocorrência de TEPT serem moderadas (Barakat, Alderfer, \& Kazak, 2006; Kazak, Boeving, Alderfer, Hwang, \& Reilly, 2005; Taïeb, Moro, Baubet, Revah-
Lévy, \& Flament, 2003).

Em pesquisa feita por Stoppelbein, Greening e Elkin (2006), um dos instrumentos utilizados para medir estresse pós-traumático foi o UCLA PTSD Reaction Index for DSM IV -Child Version, que é o mesmo adotado na presente pesquisa. Precisamente em relação ao escore total de gravidade extraído pelo instrumento foi estabelecido por estes autores que a pontuação de 12 a 24 indicaria estresse leve, de 25 a 39 apontaria estresse moderado e de 40 pontos (ou mais) sintomas graves. Além disso, 11 pontos ou menos, indicariam que a presença de sintomas de estresse pós-traumático não pode ser confirmada.

Em outro estudo feito utilizando-se o UCLA PTSD Reaction Index for DSM-IV (Pynoos, Rodriguez, Steinberg, Stuber, \& Frederick, 1998, citados por Phipps, 2007), os autores notaram que pacientes identificados como mais ansiosos reportavam significativamente mais sintomas de estresse pós-traumático do que pacientes percebidos como menos ansiosos. Entretanto, de modo geral, os resultados deste trabalho indicaram que sintomas de estresse pós-traumático não são elevados em crianças que tiveram câncer.

Schwartz e Drotar (2006) compararam um grupo de sobreviventes ao câncer infantil com um grupo controle, formado por indivíduos saudáveis, através de medidas de TEPT, depressão, qualidade de vida, humor e satisfação com a vida. Estes autores destacam que, apesar de os sobreviventes ao câncer na infância terem se mostrado bem adaptados, estão mais propensos, no futuro, a apresentarem TEPT do que o grupo controle.

Contudo, Barakat et al. (2006) alertam que as respostas pós-traumáticas não são necessariamente negativas, como, por exemplo, o surgimento de sintomas de estresse pós-traumático. Experiências traumáticas de ameaça à vida, como o câncer, poderiam resultar também em mudanças positivas para os sobreviventes e seus familiares. Ou seja, elaborar significados construtivos para o que foi vivenciado e ter novos aprendizados ampliam as possibilidades de desenvolvimento pessoal.

Em um estudo constituído por uma amostra brasileira, Delella e Araujo (2002) entrevistaram crianças (seis a 12 anos) e pais separadamente $(n=10)$. Os pais também responderam à Entrevista Clínica Estruturada - DSM IV (SCID) para avaliação de TEPT. Os resultados indicaram que as crianças demonstravam estarem mais adaptadas do que seus pais. Porém, a experiência oncológica ainda se revestia de significados negativos para pais e filhos. As autoras salientaram que a idade da criança no diagnóstico, o tipo de câncer e o tratamento realizado podem ser considerados fatores que influenciam a qualidade da sobrevivência. Sugeriram também que, a exemplo da avaliação que foi feita com os pais, futuras pesquisas estimassem a presença de TEPT infantil, possivelmente adotando o UCLA PTSD Reaction.

\section{Index for DSM IV - Child version}

Experiência parental. Conforme mencionado anteriormente, estudos voltados para a sobrevivência em oncologia contemplam a experiência parental a partir de questões específicas, como por exemplo, repercussões conjugais e familiares, identificação de sintomas de estresse pós-traumático parental e diferenças 
no enfrentamento de mães e pais. Todavia, mesmo com os conhecimentos reunidos, as pesquisas continuam a apontar dificuldades parentais. Assim, Björk, Wiebe e Hallström (2005) realizaram entrevistas com familiares de pacientes oncopediátricos, nas quais se revelaram vivências de incerteza, perda de controle pessoal, desorganização familiar e medo da morte durante o enfrentamento da enfermidade. Pöder, Ljungman e Essen (2008) insistem quanto às preocupações com estes pais, pois suas experiências são marcadas por exposições repetidas a traumas, tais como: acompanhar o filho em situações de exposição à dor, desconforto físico, depauperamento e forte dependência, além de inúmeros episódios de emergência.

Uma investigação voltada para crianças chinesas com câncer encontrou que o enfrentamento parental se caracterizou por: choque e negação; atribuição de significado para a experiência; confrontação da realidade e estabelecimento de uma nova perspectiva de vida. Estes pais chineses informaram, ainda, busca de suporte emocional, com ênfase nas relações com outros pais que também estavam no Hospital, e comprometimento com os cuidados de saúde da criança (Wong \& Chan, 2006).

Vale ressaltar que muitos trabalhos afirmam que os pais transmitem suas inquietações e angústias para seus filhos, sendo que a família tende a fazer concessões de ordem material e afetiva à criança doente. Ofertando alimentos especiais, presentes e muita atenção, os pais do paciente pediátrico acabam por ajustar o manejo educativo para compensar as restrições impostas à sua criança. Valle e Ramalho (2008) lembram que os cuidados do doente geram dúvidas e receios, sobrecarregando os pais com difíceis tarefas e comprometendo suas relações com outros filhos, inclusive pela ausência ocasionada pelas internações para exames e tratamentos.

Trask et al. (2003) comprovaram correlações positivas entre o ajustamento dos pais e o ajustamento de seus filhos adolescentes, como também no que se refere ao aumento de estratégias de enfrentamento pouco eficazes. De acordo com os autores, estratégias de esquiva do problema, caracterizadas pela negação ou inação diante de eventos estressantes, parecem ser mais incorporadas por pais e filhos do que estratégias adaptativas. Evidentemente, como se tratava de um estudo correlacional, não foi possível determinar se são os pais que influenciam os filhos, o inverso, ou se variáveis ainda pouco definidas estariam provocando tais reações. De qualquer modo, cabe lembrar que a literatura considera que as crianças aprendem comportamentos observados em seus pais, conforme ressaltaram Trask et al. (2003).

Segundo Maurice-Stam, Oort, Last e Grootenhuis (2008), os primeiros cinco anos após a alta terapêutica constituem um período significativo para ajustamento à sobrevivência, inclusive em função da diminuição de suporte social e emocional disponibilizados durante a doença. Para os autores, a coesão familiar é importante principalmente para as mães, sendo que o otimismo dos pais acerca do curso da doença relacionou-se com menor sofrimento psicológico e menos sentimentos negativos sobre a enfermidade durante a sobrevida.

No Brasil, Arrais e Araujo (1999) entrevistaram 10 crianças sobreviventes, com idades variando entre seis e 12 anos, e seus pais. As crianças também responderam o Inventário de Ansiedade Traço-Estado para Crianças (IDATE-C) e o Inventário de Depressão Infantil (CDI). Mais da metade das crianças apresentou-se bem adaptada e não revelou altos índices de depressão e ansiedade. Contudo, seus pais sofriam forte apreensão e ansiedade, as quais foram relatadas em entrevistas semiestruturadas realizadas no contexto domiciliar.

Na pesquisa nacional conduzida por Delella e Araujo (2002), foi possível verificar que o enfrentamento dos pais caracterizavase pela busca de informação, ajuda espiritual e suporte social. Para eles, a disponibilidade de suporte social foi considerada fator relevante para o enfrentamento. Segundo as autoras, os filhos pareceram adotar estilos semelhantes aos de seus pais, mas não foi possível estabelecer relação entre as atitudes da criança e as de seus pais.

Em síntese, de um lado é importante considerar os avanços alcançados pelos estudos internacionais em Psico-Oncologia Pediátrica, notadamente no que se refere à problemática da sobrevivência ao câncer na infância, os quais suscitam interesses similares no contexto brasileiro. De outro lado, os estudos nacionais já realizados - tanto com crianças como com pais, a partir de diferentes enfoques metodológicos - confirmam a relevância clínica e científica em se aprimorar os conhecimentos até hoje conquistados. Por exemplo, é preciso conhecer a percepção que os filhos têm sobre a experiência parental, uma vez que os trabalhos focalizam a visão dos pais sobre seus filhos. Também são necessárias mais investigações sobre o transtorno do estresse pós-traumático entre crianças, em particular no contexto da saúde.

Assim sendo, realizou-se uma pesquisa visando analisar a experiência da sobrevivência ao câncer na infância, enfatizandose a avaliação de TEPT infantil e a percepção da criança sobre a experiência parental. Mais especificamente, estabeleceram-se os seguintes objetivos: a) identificar a ocorrência de um quadro de TEPT ou de alguns de seus sintomas em crianças sobreviventes ao câncer; b) investigar a percepção da criança sobrevivente acerca da experiência de seus pais; c) analisar, em conjunto, as taxas de TEPT com os relatos das crianças sobre seus pais.

\section{Método}

A partir de uma abordagem multimetodológica - a qual envolveu a aplicação de diversos instrumentos para análise quantitativa e qualitativa - desenvolveu-se uma pesquisa de natureza descritiva e exploratória.

\section{Participantes}

Em conformidade com a literatura especializada, foi adotado como critério de inclusão o período de um ano, no mínimo, fora de tratamento para considerar o participante como sobrevivente (Barakat et al., 2006; Kazak et al., 2004). Com relação à idade, utilizou-se o critério de, no mínimo, cinco anos de idade durante mais da metade da duração da doença. Isso porque muitas pesquisas têm mostrado que a partir dessa faixa etária é maior a suscetibilidade ao estresse pós-traumático (Barakat et al., 2006). Assim sendo, preencheram esses critérios, e concordaram em participar desta pesquisa, 15 crianças - oito meninos e sete meninas, com escolaridade entre $3^{-}$e $7^{\circ}$ ano do 
ensino fundamental - na faixa etária de oito a 12 anos $(M=9,9$ anos de idade). No momento do diagnóstico da doença, 11 dos participantes já tinham cinco anos ou mais, com variação de três a sete anos. A idade no término do tratamento variou de cinco a oito anos, sendo que 11 sobreviventes encontravam-se na faixa dos seis aos sete anos de idade. O tempo mínimo de tratamento foi de três meses e o máximo de três anos e três meses, com média de 18 meses. Dez crianças já haviam encerrado o tratamento há três anos ou mais. Quanto às enfermidades, a distribuição foi a seguinte: leucemia linfoide aguda $(n=4)$, linfomas $(n=3)$, leucemia mieloide aguda $(n=1)$, leucemia mieloide crônica $(n$ $=1)$, tumor de Wilms $(n=1)$, tumor neuroectodérmico primitivo $(n=2)$, rabdmiossarcoma $(n=2)$ e astrocitoma $(n=1)$.

\section{Instrumentos}

1) questionário, respondido pelos pais, para obter dados sociodemográficos sobre a criança e sua família, além de corroborar as informações coletadas a partir dos prontuários.

2) jogo de tabuleiro empregado para facilitar o estabelecimento de rapport entre a pesquisadora e a criança.

3) roteiro de entrevista semiestruturada, elaborado pela pesquisadora com base em estudos sobre o tema, para avaliar a percepção das crianças sobreviventes sobre a experiência dos pais, examinando atitudes e crenças individuais. Era composto pelos seguintes eixos temáticos: fase do diagnóstico, fase do tratamento, organização familiar, fase da alta e fase da sobrevivência. Antes de iniciar a coleta de dados, o roteiro foi testado e aprimorado.

4) brinquedo de encaixe (Playmobil hospitalar), o qual foi utilizado para facilitar o início da abordagem do tema da entrevista.

5) UCLA PTSD Reaction Index for DSM IV (Revision 1) Child Version (Rodriguez, Steinberg, \& Pynoos, 1999), criado pelo Trauma Psychiatry Service na University of California at Los Angeles, o qual autorizou o uso deste instrumento nesta pesquisa. Trata-se de um instrumento que identifica, de maneira breve, sintomas de TEPT em crianças que vivenciaram eventos traumáticos, fornecendo também informações sobre a frequência desses sintomas (Steinberg, Brymer, Decker, \& Pynoos, 2004; Stoppelbein et al., 2006). Este instrumento oferece também informações preliminares sobre um possível diagnóstico de TEPT. A versão infantil é adequada para a faixa etária de sete a 12 anos. Para a presente pesquisa, foram feitas a tradução reversa por pesquisadores bilíngues e a adaptação do instrumento, de acordo com o conhecimento clínico e a experiência do grupo de pesquisa quanto a este tema.

Vale esclarecer que os itens 1 a 13 do referido instrumento, fazem uma triagem acerca da exposição a eventos traumáticos, que deve ser respondida com sim ou não. No item 14, é questionado quando o evento ocorreu e é solicitada uma breve descrição sobre ele. Os itens 15 a 21 investigam sobre os critérios "A1" de TEPT do DSM-IV com relação a aspectos do evento traumático e os itens de 22 a 26 tratam dos critérios "A2", indagando sobre a experiência subjetiva da criança durante ou após o trauma vivenciado. Já o item 27 investiga uma reação dissociativa por ocasião do evento traumático, sendo que, nos itens de 15 a 27, a criança deve responder sim ou não. No final do instrumento, existem 20 itens que questionam sobre a frequência de sintomas de estresse pós-traumático e outros aspectos relacionados, sendo que a criança deve responder por meio de uma escala (nunca, pouco, às vezes, quase sempre e sempre). Um diagnóstico de TEPT é estabelecido, quando são preenchidos todos os critérios: A (Evento traumático), B (Reexperimentar), C (Evitação) e D (Hiperestimulação). É possível elaborar um diagnóstico parcial, nos casos em que é contemplado o critério $\mathrm{A}$, adicionado de mais dois outros critérios $(\mathrm{B}+\mathrm{C}$ ou $\mathrm{B}+\mathrm{D}$ ou $\mathrm{C}+\mathrm{D})$. Estudos recentes internacionais já comprovaram a validade psicométrica deste instrumento e sua consistência interna (Stoppelbein et al., 2006, Steinberg et al., 2004). Mas, no Brasil, ele ainda não foi validado.

\section{Procedimentos para coleta de dados}

O primeiro contato com os sobreviventes e seus responsáveis foi feito no ambulatório da Unidade de Onco-Hematologia Pediátrica de um Hospital, no dia de atendimento exclusivo a pacientes que finalizaram o tratamento e continuam sendo acompanhados no serviço. Após uma breve exposição sobre a investigação, solicitou-se indicação de contato telefônico para agendamento de uma visita domiciliar. Logo no início dessa visita, enquanto a pesquisadora utilizava o jogo de tabuleiro com a criança (eventualmente também com seus irmãos), em outro ambiente, uma auxiliar de pesquisa apresentava o Termo de Consentimento Livre e Esclarecido e aplicava o questionário sociodemográfico a um dos pais. Em seguida, a auxiliar integrava-se à situação lúdica. Terminado o jogo, a pesquisadora e sua auxiliar entrevistavam a criança. Os relatos foram registrados em áudio após o consentimento do participante. Por fim, aplicava-se o UCLA PTSD Reaction Index for DSM IV - Child Version.

\section{Procedimentos de análise dos dados}

Seguiram-se as recomendações previstas no manual do UCLA PTSD Reaction Index for DSM IV - Child Version para a análise dos dados obtidos, encontrando-se as pontuações em cada critério e no total (escore de gravidade) para TEPT. Adotou-se também o software SPSS 17.0 para correlacionar as variáveis clínicas (idade no diagnóstico, duração da doença, idade ao final do tratamento e tempo fora de tratamento) com esses escores encontrados. Todas as entrevistas foram transcritas e o material registrado foi submetido à análise de conteúdo temática (Bardin, 1977). Assim, a partir da literatura especializada, mencionada anteriormente, e dos relatos dos participantes, definiram-se as seguintes categorias: a) percepção do paciente sobre mudanças parentais após a experiência oncológica; b) impacto da doença e do tratamento nas rotinas profissional e familiar dos pais; c) percepção da criança sobre o enfrentamento parental; d) percepção sobre cuidados provenientes dos pais durante a doença; e) experiência parental durante a fase da sobrevivência. $\mathrm{Na}$ fase seguinte, cada categoria relativa à experiência parental foi comparada com os resultados do escore total de gravidade extraído do UCLA PTSD Reaction Index for DSM IV - Child Version.

\section{Resultados e discussão}




\section{Transtorno de estresse pós-traumático infantil}

A Figura 1 apresenta os escores de gravidade calculados pela aplicação do UCLA PTSD Reaction Index for DSM IVChild Version, cuja variação foi de 7 a 45 (a pontuação máxima de escore de gravidade é 68). Nas instruções de análise do instrumento, não são descritos valores médios para o escore total de gravidade, uma vez que se ressalta apenas que quanto maior o número de sintomas, maior a gravidade do estresse pós-traumático. A média foi de 25,06 e a maioria das crianças $(n=9)$ apresentou escores entre 11 e 30. Considerando-se o critério estipulado por Stoppelbein et al. (2006) com amostras internacionais, na presente pesquisa, seis participantes apresentaram estresse leve, sete indicaram estresse moderado e apenas um revelou sintomas graves.

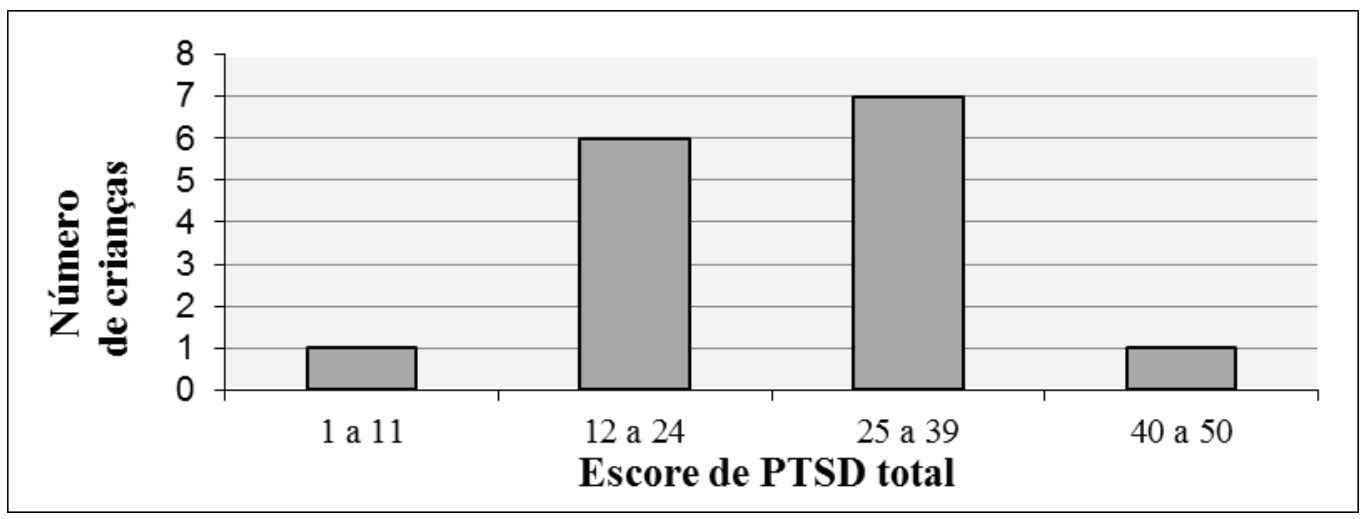

Figura 1

Escore total de gravidade de transtorno de estresse pós-traumático (PTSD Severity Score).

Cabe explicitar que, segundo o instrumento utilizado, para preencher o Critério B de TEPT seria necessário apenas um sintoma, para o Critério C no mínimo três sintomas e para o Critério D no mínimo dois. É importante ressaltar que, cada um dos últimos 20 itens correspondia a um sintoma e que as pontuações 3 ou 4 foram definidas como o mínimo para se caracterizar a presença deste, que se referiam a quase sempre ou sempre na escala utilizada. Com relação ao Critério A, 12 crianças preencheram todos os requisitos, que estão relacionados a aspectos do evento traumático propriamente dito e da experiência subjetiva durante sua ocorrência ou após seu término. Cinco participantes preencheram os requisitos para o Critério B, cinco para o Critério $\mathrm{C}$ e quatro para o Critério D. Entretanto, é preciso esclarecer que, em conformidade com a literatura, determinou-se como pontuação mínima para cada sintoma o nível 3, que se referia a "quase sempre" ou "sempre" no instrumento.

Um possível diagnóstico de TEPT foi evidenciado em apenas um dos participantes e o diagnóstico parcial em quatro deles. Mas, as instruções contidas no manual de aplicação do instrumento enfatizam que o diagnóstico deve ser confirmado a partir de avaliações clínicas e que a pontuação obtida em cada critério é um fator importante na confirmação ou não do diagnóstico. De qualquer modo, os dados corroboram as taxas moderadas já comunicadas em estudos internacionais (Barakat et al., 2006; Kazak et al., 2004; Pynoos et al., 1998, citados por Phipps, 2007).

Os principais resultados da correlação bivariada de Pearson para as variáveis clínicas e os escores obtidos no instrumento demonstraram que três variáveis se correlacionaram com as pontuações do UCLA PTSD Reaction Index for DSM IV-Child Version, a saber:

- idade na época do diagnóstico: correlação positiva com diagnóstico parcial de TEPT $(r=0,519, p=0,05) \mathrm{e}$ preenchimento dos requisitos para o Critério $\mathrm{D}(r=0,519, p$ $=0,05)$;

- duração da doença: correlação negativa com diagnóstico parcial $(r=-0,544, p=0,05)$;

- idade ao final do tratamento: correlação negativa com o escore no Critério B $(r=-0,456, p=0,05)$.

A variável "tempo fora de tratamento" não se correlacionou significativamente com os escores do instrumento. O estabelecimento de um diagnóstico parcial de TEPT foi mais evidente entre as crianças com mais idade por ocasião do diagnóstico do câncer, o que permite presumir que estas estariam mais suscetíveis às consequências negativas desta experiência. Este resultado reforça a posição de Barakat et al. (2006) quanto à adoção do critério de cinco a sete anos de idade na época do diagnóstico para constituição das amostras, pois muitas pesquisas têm mostrado que a partir dessa faixa etária é maior a suscetibilidade ao estresse pós-traumático.

Quanto à correlação entre o diagnóstico parcial e a duração da doença, observou-se que o TEPT foi mais identificado entre aqueles participantes que vivenciaram um período menor de duração da enfermidade. Uma possível explicação seria que, embora o tratamento fosse curto, também foi mais intenso e, consequentemente, vivenciado de maneira mais traumática. Além disso, crianças que receberam um tratamento prolongado podem ter tido mais tempo para aceitar e melhor compreender suas experiências, o que atenuaria as repercussões traumáticas. Por fim, a correlação negativa entre a idade ao final do tratamento e a pontuação do Critério B também parece apontar maior suscetibilidade das crianças com mais idade.

É importante mencionar que não foram evidenciadas diferenças significativas entre os diferentes tipos de câncer. Tratase, possivelmente, de uma decorrência do tamanho reduzido da 
amostra obtida.

\section{Entrevistas com as crianças acerca da experiência parental}

Percepção do paciente sobre mudanças parentais após a experiência oncológica. Somando-se aqueles que relataram mudanças em padrões de comportamento com os que mencionaram mudanças, mas não explicaram, não lembraram ou não souberam dizer o que efetivamente mudou, 13 participantes relataram que os pais apresentaram alguma mudança após a experiência traumática. Ou seja, a maioria percebe mudanças em seus pais, mas não é capaz de especificá-las. Tais constatações podem resultar da influência do contexto de entrevista; do repertório verbal da criança; e também do tipo de interação entre pais e filhos, na qual se estimule, ou não, a expressão da criança quanto às suas percepções. Apesar das dificuldades encontradas para se obter seus relatos, reiteram-se os argumentos de Björk et al. (2005) quanto à importância de se conhecer a percepção infantil acerca de suas próprias experiências e de sua família, designando-lhes um papel ativo no processo investigativo.

Salienta-se que, no total, sete participantes mencionaram especificamente mudanças comportamentais em seus pais, como por exemplo: busca e aquisição de informações relativas à doença e medicamentos e desenvolvimento de distúrbios. Também informaram aumento de comportamentos de cuidado e atenção e mudanças na expressão da afetividade em relação ao filho que esteve doente. Estes resultados fortalecem observações de trabalhos anteriores (Arrais \& Araujo, 1999; Delella \& Araujo, 2002; Espíndula, 2001). Ainda considerando este grupo que relatou mudanças comportamentais, as crianças também perceberam mudanças positivas na vida de seus pais, salientando, por exemplo, diminuição de conflitos conjugais, maior afetividade com os filhos e aumento de comportamentos de cuidados, atenção e interação com elas. Esta percepção reafirma os benefícios e mudanças positivas resultantes do enfrentamento de situações adversas, tal como defenderam Barakat et al. (2006), Janoff-Bulman (1999) e Lopes (2001).

Impacto da doença e do tratamento nas rotinas profissional e familiar dos pais. Esta categorização diz respeito ao impacto da enfermidade e dos cuidados médicos na vida dos pais, relatado pela criança já na fase de sobrevida. Apenas uma das crianças relatou a presença tanto de suporte social emocional como de apoio instrumental, enquanto as demais $(n=12)$ citaram que apenas um deles estava presente para seus pais. Outras duas crianças não mencionaram suporte para os pais. A existência ou não de suporte social foi considerada pelos participantes como fator de impacto na maneira como o câncer foi enfrentado por seus cuidadores, concentrando a maior frequência de relatos. O suporte social é destacado por muitos autores (Cunha \& Rúmen, 2008; Straub, 2007; Valle \& Ramalho, 2008). Cabe comentar, inclusive, que Delella e Araujo (2002) verificaram, a partir dos relatos de pais de sobreviventes, que o suporte social é fator influente no enfrentamento da doença. E, sendo assim, a percepção das crianças sobre seus pais, delineada pela presente pesquisa, confirma os relatos feitos pelos pais no trabalho destas autoras. No que tange a mudanças na dinâmica familiar, as crianças realçaram a necessidade de seus pais delegarem os cuidados dos irmãos saudáveis para outras pessoas como a principal modificação. A preocupação dos pais com os filhos sadios também foi bastante citada, sugerindo que, para os sobreviventes, dificuldades e mudanças relacionadas aos cuidados de seus irmãos tiveram impacto na vida de seus pais.

Percepção da criança sobre o enfrentamento parental. Em relação à distinção, corrente na literatura especializada, sobre enfrentamento focalizado no problema ou focalizado na emoção (Gimenes, 1997), verificou-se no presente estudo baixa frequência de relatos das crianças sobre enfrentamento focalizado no problema por parte de seus pais. Contrariamente a Delella e Araujo (2002), a modalidade predominante reconhecida pelas crianças - foi o enfrentamento focalizado na emoção, caracterizado por choro e lágrimas como expressão de tristeza, medo e incerteza. É possível supor, portanto, que pais e filhos têm percepções diferentes sobre a experiência parental.

Ademais, para algumas destas crianças, seus pais utilizavam estratégias como negação (recusa de seus pais em acreditar na existência da doença, caracterizada por comportamentos de esquiva de pensamentos e sentimentos relacionados à situação aversiva e por descrédito em informações médicas), culpabilização de outros, não expressão de sentimentos e omissão de informações sobre a enfermidade para o participante, cuja inadequação pode se refletir na qualidade de vida após a cura (Arrais \& Araujo, 1999; Silva, Teles, \& Valle, 2005). Chama a atenção que a criança que obteve o maior escore $(n=45)$ no UCLA PTSD Reaction Index for DSM IV - Child Version e manifestou oito sintomas, vinculou todos esses comportamentos a seu pai. Assim, no caso deste participante, o modo de enfrentamento parental parece estar relacionado com a presença de PTSD e sintomas.

Percepção sobre os cuidados parentais durante a doença. Os participantes relataram com maior frequência que os cuidados fornecidos por seus pais envolviam "dar coisas" e "fazer coisas" para o filho doente, coincidindo com os comentários de Valle e Ramalho (2008) sobre concessões materiais durante a enfermidade. As crianças perceberam ainda maior atenção, afetividade e proibições relacionadas a determinadas brincadeiras e ida a certos locais julgados como de risco. Desobediências passaram a não serem repreendidas com punição física.

Experiência parental durante a fase da sobrevivência. Praticamente o mesmo número de crianças mencionou comportamentos de esquiva e falta de verbalizações sobre a doença $(n=10)$, bem como ausência de comportamentos de esquiva ( $n=12$ ), sendo que sete entrevistados tiveram ambos os tipos de relato. Uma análise mais cuidadosa revela a complexidade desta evidência: em certas situações, os pais se esquivam ou não comentam, em outras fazem o contrário. É importante esclarecer que as crianças que relataram dificuldades, por parte dos pais, em falar sobre a experiência oncológica, quando o tema é abordado pelo sobrevivente, também salientaram que os pais conseguem fazê-lo com amigos e familiares $(n=4)$. Contudo, para se saber se estas atitudes constituem indicadores de risco para estresse pós- 
traumático é necessário realizar pesquisas de natureza clínica.

De modo geral, as crianças são capazes de perceber o quanto o contexto hospitalar é aversivo para seus cuidadores. Algumas disseram que ao irem ao hospital, seus pais se lembram da fase da doença e do tratamento, mostrando-se tristes ou chorando ( $n$ =7). De acordo com Baum e Spencer (1997, conforme citado em Marks, Murray, Evans, \& Willig, 2000), a sensibilidade a novos eventos estressantes é um dos sintomas de TEPT. Para Janoff-Bulman (1999), as lembranças intrusivas - tais como as que foram relatadas nas entrevistas - impõem a revivência do trauma, o que indicaria a necessidade da experiência ainda ser elaborada e integrada. Neste sentido, vale retomar os dados obtidos por Delella e Araujo (2002), os quais apontaram que o retorno ao hospital é visto com um evento gerador de forte ansiedade pelos próprios pais.

Comparação entre os resultados no TEPT infantil e a percepção da criança sobre a experiência parental. Devese destacar o relato da única criança que obteve um possível diagnóstico de TEPT e o maior escore total de gravidade. Segundo esta menina, seus pais não falavam sobre a experiência oncológica com outras pessoas, mas conversavam com ela algumas vezes. Esta mesma criança obteve sua maior pontuação no Critério $\mathrm{C}$, que envolve a esquiva de situações relacionadas ao evento traumático. Esta congruência entre criança e pais remete aos comentários de Trask et al. (2003), reportados anteriormente, sobre a analogia de atitudes e comportamentos entre eles.

O contexto hospitalar foi descrito como suscitando reações negativas por seis participantes, que foram aqueles que obtiveram as cinco maiores pontuações para diagnóstico de TEPT. Este resultado converge com os relatos dos pais, levantados por Delella e Araujo (2002), sobre a ansiedade que sentem no retorno ao hospital para exames de rotina do filho. Vivências paradoxais de recidiva versus cura, por parte dos pais, também puderam ser reconhecidas em duas destas entrevistas, o que sugere alguma relação com o estresse pós-traumático da criança.

\section{Considerações finais}

Identificaram-se taxas moderadas de diagnóstico parcial e total de TEPT. Além disso, as taxas de sintomas de estresse pós-traumático também foram baixas, uma vez que na presente investigação, em um total de 17 sintomas, a média de sintomatologia foi de 3,6 e, no máximo, de oito sintomas para um dos participantes. As correlações entre as variáveis clínicas e os escores do UCLA PTSD Reaction Index for DSM IV Child Version mostraram que a idade da criança, por ocasião do diagnóstico de câncer, correlaciona-se positivamente com o diagnóstico parcial de TEPT, ao passo que a duração da doença correlaciona-se negativamente. Todavia, é preciso prudência na interpretação destes dados, em razão do tamanho limitado da amostra investigada.

Ressalta-se que o instrumento revela qualidade clínica para profissionais e serviços que realizam o acompanhamento de pacientes sobreviventes ao câncer na infância. Porém, ainda são necessários estudos de validação para a população brasileira.

A percepção das crianças acerca da experiência parental corroborou a literatura especializada, inclusive no que se refere às investigações que abordaram as percepções dos pais sobre suas próprias experiências. Ao que parece, existe relação entre o modo como a experiência dos pais é percebida por seus filhos e o escore total de gravidade de TEPT infantil. Todavia, como as crianças com maiores pontuações de escore total de gravidade expressaram ambiguidades e contradições em suas falas, é indispensável ampliar as pesquisas para melhor compreender a vivência paradoxal que caracteriza a sobrevivência (Arrais \& Araujo, 1999; Espíndula, 2001; Silva et al., 2005).

Sugere-se que futuras pesquisas ampliem o número de participantes, no intuito de assegurar generalização dos dados e refinamento do instrumento UCLA PTSD Reaction Index for DSM IV - Child Version para a população brasileira. Neste mesmo sentido, é crucial discriminar entre os diversos tipos de câncer e seus respectivos tratamentos. Também são indispensáveis estudos longitudinais com medidas sistemáticas deste transtorno. Detectar a relação entre diagnóstico parcial e total de TEPT ao longo das fases de desenvolvimento do sobrevivente - e especialmente em seus períodos de transição constituirá outra contribuição significativa em Psico-Oncologia Pediátrica.

\section{Referências}

Alapetite, C., Baillet, F., Champemond, S., Dessard-Diana, B., Housset, M. \& Michel-Langlet, P. (1988). Opposition pour le malade cancéreux entre notion de guérison et surveillance prolongée. Psychologie Médicale, 20(9), 1283-1284.

Alby, N. (1988). Difficulté pour les parents d'intégrer la notion de guérison: conséquences à long terme de la maladie sur les familles. Psychologie Médicale, 20(9), 1350-1351.

Alderfer, M., Labay, L., \& Kazak, A. E. (2003). Brief report: does posttraumatic stress apply to siblings of childhood cancer survivors? Journal of Pediatric Psychology, 28(4), 281-286.

American Psychiatric Association (2000). Manual Diagnóstico e Estatístico de Transtornos Mentais. DSM-IV (4⿳⺈冂a . ed.). Porto Alegre: Artmed.

Andrea, M. L. M. (2008). Oncologia pediátrica. In V. A. Carvalho et al. (Orgs.), Temas em Psico-Oncologia (pp. 477-495). São Paulo: Summus Editorial.

Araujo, T. C. C. F., \& Arrais, A. R. (1998). A sobrevivência em oncologia. uma vivência paradoxal. Psicologia Ciência e Profissão, 18(2), 2-9. Recuperado de http://www.bvs-psi.org.br.

Arrais, A. R., \& Araujo, T. C. C. F. (1999). Recidiva versus cura: a vivência paradoxal da sobrevivência ao câncer na infância. Revista Brasileira de Cancerologia, 45(3), 15-22.

Barakat, L. P., Alderfer, M. A., \& Kazak, A. E. (2006). Posttraumatic growth in adolescent survivors of cancer and their mothers and fathers. Journal of Pediatric Psychology, 31(4), 413-419.

Bardin, L. (1977). L'analyse de contenu. Paris: Presses Universitaires de France.

Baumeister, R. F., Faber, J. E., \& Wallace, H. M. (1999). Coping and ego depletion. recovery after the coping process. In C. R. Snyder (Org.), Coping: the psychology of what works (pp. 50-69). Nova Iorque: Oxford University Press.

Björk, M., Wiebe T., \& Hallström, I. (2005). Striving to survive: families' lived experiences when a child is diagnosed with cancer. Journal of Pediatric Oncology Nursing, 22(5), 265-275.

Braga, P. E., Latorre, M. R. D. O., \& Curado, M. P. (2002). Câncer na infância: uma análise comparativa da incidência, mortalidade e sobrevida em Goiânia (Brasil) e outros países. Cadernos de Saúde Pública, 18(1), 33-44.

Cunha, A. D., \& Rúmen, F. A. (2008). Reabilitação psicossocial do paciente com câncer. In V. A. Carvalho et al. (Orgs.), Temas em Psico-Oncologia (pp. 
505-516). São Paulo: Summus Editorial.

Coleman, M. P., Quaresma, M., Berrino, F., Lutz, J., Angelis, R., Capocaccia, R.,... Group, C., W. (2008). Cancer survival in five continents: a worldwide population-based study (CONCORD). Lancet Oncology, 9(8), 730-756. doi:10.1016/S1470-2045(08)70179-7

Comitê Pediátrico da Sociedade Brasileira de Psico-Oncologia (2000). Orientações psicossociais em Oncologia Pediátrica. (L. P. C. Françoso \& E. R. M. Valle, Trads.). Recuperado de http:// www.sbpo.org.br/images/ pdf/siop_2000.pdf.

Dahlquist, L. M., Czyzewski, D. I., \& Jones, C. L. (1996). Parents of children with cancer: a longitudinal study of emotional distress, coping style, and marital adjustement two and twenty months after diagnosis. Journal of Pediatric Psychology, 21(4), 541-554.

Delella, L. A., \& Araujo, T. C. C. F. (2002). Câncer na infância: uma investigação sobre a avaliação desordem de estresse pós-traumático parental e a experiência da sobrevivência. Psicologia e Argumento, 20(31), 42-48.

Eiser, C., \& Havermans, T. (1994). Long term social adjustment after treatment for childhood cancer. Archives of Disease in Childhood, 70(1), 66-70.

Espíndula, J. A. (2001). Vivências de mães em situação de recidiva de câncer. In E. R. M. Valle (Org.), Psico-oncologia pediátrica (pp. 129-179). São Paulo: Casa do Psicólogo.

Figueira, I., \& Mendlowicz, M. (2003). Diagnóstico do transtorno de estresse pós-traumático. Revista Brasileira de Psiquiatria, 25, 12-16.

Frisch, G. A., \& Desmarez C. (1988). Séquelles psychologiques et cancer chez l'enfant en rémission. Psychologie Médicale, 20(9), 1343-1344.

Gimenes, M. G. G. (1997). A teoria do enfrentamento e suas implicações para sucessos e insucessos em Psiconcologia. In M. G. G. Gimenes \& M. H. Fávero (Orgs.), A mulher e o câncer (111-147). São Paulo: Editorial Psy.

Janoff-Bulman, R. (1999). Rebuilding shattered assumptions after traumatic life events. Coping processes and outcomes. In C. R. Snyder (Org.), Coping: The psychology of what works (pp. 305-323). Nova Iorque: Oxford University Press.

Kazak, A. E., Boeving, C. A., Alderfer, M. A., Hwang, T., \& Reilly, A. (2005). Posttraumatic Stress Symptoms during treatment in parents of children with cancer. Journal of Clinical Oncology, 23(30), 7405-7410.

Kazak, A. E., Alderfer, M., Rourke, M., Simms, S., Streisand, R., \& Grossman, J. R. (2004). Posttraumatic Stress Disorder (PTSD) and Posttraumatic Stress Symptoms (PTSS) in families of adolescent childhood cancer survivors. Journal of Pediatric Psychology, 29(3), 211-219.

Lopes, D. P. L. O. (2001). A organização familiar e o acontecer do tratamento da criança. In E. R. M. Valle (Org.), Psico-oncologia pediátrica (pp. 13-74). São Paulo: Casa do Psicólogo.

Lozowski, S. L. (1993). Views of childhood cancer survivors - selected perspectives. Cancer Supplement, 71(10), 3354-3357.

Marks, D. F., Murray, M., Evans, B., \& Willig, C. (2000). Health Psychology. Londres: Sage.

Maurice-Stam, H., Oort, F. J., Last, B. F., \& Grootenhuis, M. A. (2008). Emotional functioning of parents of children with cancer: the first years of continuous remission after the end of treatment. Psycho-Oncology, 17, 448-459.

Mulhern, R. K., Fairclough, D. L., Smith, B., \& Douglas, S. M. (1992). Maternal depression, assessment methods, and physical symptoms affect estimates of depressive symptomatology among children with cancer. Journal of Pediatric Psychology, 17(3), 313-326.

Nicholson, H. S., Fears, T., \& Byrne, J. (1994). Death during adulthood in survivors of childhood and adolescent cancer. Cancer, 73(12), 3094-3102.

Perina, E. M., Mastellaro, M. J., \& Nucci, N. A. G. (2008). Efeitos tardios do tratamento do câncer na infância e na adolescência. In V. A. Carvalho et al. (Orgs.), Temas em Psico-Oncologia (pp. 496-504). São Paulo: Summus Editorial.

Phipps, S. (2007). Adaptive style in children with cancer: implications for a positive psychology approach. Journal of Pediatric Psychology, 32(9), 1055-1066.

Pöder, U., Ljungman, G., \& Essen, L. (2008). Posttraumatic stress disorder among parents of children on cancer treatment: a longitudinal study. PsychoOncology, 17, 430-437.

Pucheu, S. (1988). Le sentiment de guérison et ses aléas psychiques. Psychologie Médicale, 20(9), 1279-1280.

Resende, T. I. M., \& Araujo, T. C. C. F. (1999). Relacionamento mãe-criança com câncer: a importância da díade afetiva. Psico, 30(1), 51-65.

Rodriguez, N., Steinberg, A., \& Pynoos, R. S. (1999). UCLA PTSD Index for DSM-IV instrument information: Child version, parent version, adolescent version. Los Angeles: UCLA Trauma Psychiatry Services.

Roth, A. J., Holland, J. C., \& Murillo, M. (2006). Aspectos psicológicos de las enfermedades hematológicas malignas. In A. A. Prada (Org.), Manual de Psicooncología (pp. 143-171). Bogotá: Javegraf.

Schwartz, L., \& Drotar, D. (2006). Posttraumatic stress and related impairment in survivors of childhood cancer in early adulthood compared to health peers. Journal of Pediatric Psychology, 31(4), 356-366.

Silva, G. M., Teles, S. S., \& Valle, E. R. M. (2005). Estudo sobre as publicações brasileiras relacionadas a aspectos psicossociais do câncer infantil-período de 1998 a 2004. Revista Brasileira de Cancerologia, 51(3), 253-261.

Steinberg, A. M., Brymer, M. J., Decker, K. B., \& Pynoos, R. S. (2004). The University of California at Los Angeles Post-traumatic Stress Disorder Reaction Index. Current Psychiatry Reports, 6(2), 96-100.

Stoppelbein, L. A., Greening, L., \& Elkin, T. D. (2006). Risk of posttraumatic stress symptoms: a comparison of child survivors of pediatric cancer and parental bereavement. Journal of Pediatric Psychology, 31(4), 367-376.

Straub, R. O. (2007). Health psychology: A biopsychosocial approach ( $\left.2^{\text {nd }} \mathrm{ed}.\right)$. Nova Iorque: Worth Publishers.

Taïeb, O., Moro, M., Baubet, T., Revah-Lévy, A., \& Flament, M. (2003). Posttraumatic stress symptoms after childhood cancer. European Child \& Adolescent Psychiatry, 12(6), 255-264.

Trask, P. C., Paterson, A. G., Trask, C. L., Bares, C. B., Birt, J., \& Maan, C. (2003). Parent and adolescent adjustment to pediatric cancer: associations with coping, social support, and family function. Journal of Pediatric Oncology Nursing, 20(1), 36-47.

Valle, E. R. M., \& Ramalho, M. A. N. (2008). O câncer na criança: a difícil trajetória. In V. A. Carvalho et al., (Orgs.), Temas em Psico-Oncologia (pp. 505-516). São Paulo: Summus Editorial.

Wong, M. Y., \& Chan, S. W. (2006). The qualitative experience of Chinese parents with children diagnosed of cancer. Journal of Clinical Nursing, 15, 710-717.

Camila Tokarski Boaventura, mestre em Processos de Desenvolvimento Humano e Saúde/ Psicologia pela Universidade de Brasília, é psicóloga do Instituto Nacional de Câncer. Endereço para correspondência: Rua Visconde de Ouro Preto,40, ap. 802, Botafogo, Rio de Janeiro, CEP 22250-180. Telefones: (21) 8118-7991 e (21) 3268-3348. Email:camilaboaventura@gmail.com Tereza Cristina Cavalcanti Ferreira de Araujo, pós-doutora pela Unesco (França) e doutora pela Université de Paris X-Nanterre, é professora Associada 3 na Universidade de Brasília. E-mail: araujotc@unb.br 